\title{
The Use of Fractal Dimension Analysis in Estimation of Blood Vessels Shape in Transplantable Mammary Adenocarcinoma in Wistar Rats after Photodynamic Therapy Combined with Cysteine Protease Inhibitors
}

\author{
Kamil Jurczyszyn, ${ }^{1}$ Beata J. Osiecka, ${ }^{2}$ and Piotr Ziółkowski ${ }^{2}$ \\ ${ }^{1}$ Department of Dental Surgery, Wroclaw Medical University, Krakowska 26, 50-425 Wroclaw, Poland \\ ${ }^{2}$ Department of Pathomorphology, Wroclaw Medical University, Marcinkowskiego 1, 50-368 Wrocław, Poland \\ Correspondence should be addressed to Kamil Jurczyszyn, kjurczysz@interia.pl
}

Received 26 June 2012; Accepted 1 August 2012

Academic Editor: Facundo Ballester

Copyright () 2012 Kamil Jurczyszyn et al. This is an open access article distributed under the Creative Commons Attribution License, which permits unrestricted use, distribution, and reproduction in any medium, provided the original work is properly cited.

Fractal dimension analysis (FDA) is modern mathematical method widely used to describing of complex and chaotic shapes when classic methods fail. The main aim of this study was evaluating the influence of photodynamic therapy (PDT) with cystein proteases inhibitors (CPI) on the number and morphology of blood vessels inside tumor and on increase of effectiveness of combined therapy in contrast to PDT and CPI used separately. Animals were divided into four groups: control, treated using only PDT, treated using only CPI and treated using combined therapy, PDT and CPI. Results showed that time of animal survival and depth of necrosis inside tumor were significantly higher in CPI+PDT group in contrast to other groups. The higher value of fractal dimension (FD) was observed in control group, while the lowest value was found in the group which was treated by cystein protease inhibitors. The differences between FD were observed in CPI group and PDT+CPI group in comparison to control group. Our results revealed that fractal dimension analysis is a very useful tool in estimating differences between irregular shapes like blood vessels in PDT treated tumors. Thus, the implementation of FDA algorithms could be useful method in evaluating the efficacy of PDT.

\section{Introduction}

One of the important factors responsible for the tumor growth is blood supply. Many studies revealed that the anatomic and spatial structure of tumor blood vessels is more chaotic than that of the normal tissues. One of the classic ways of analyzing the blood vessels is direct count in the light microscope. Immunohistochemical staining is very useful against specific proteins within blood vessel wall, for example vascular endothelial growth factor (VEGF), basic fibroblast growth factor (bFGF), cluster of differentiation such as CD34, and others $[1,2]$. Many studies show that PDT is responsible for overexpression of VEGF in vivo and in vitro [3-5]. However, in the case of such complex and chaotic shapes of tumor blood vessels classic methods of evaluation may fail. Fractal dimension analysis may be very helpful in these cases. Fractal is a shape which is described by potentially simple mathematic formulas. If these formulas are iterated into infinity they may create shapes which we are able of magnifying without the end and each time we can see infinity numbers of shape details - it is a self similarity feature. Classic examples of fractals are Cantor's set, Koch's snowflake, and Sierpinski triangle (see Figure 1).

Many natural shapes are very similar to fractals, for example, coastline, trees, clouds, bronchial tree, and blood vessels network. Any above-mentioned structures poses main feature of fractal-self-similarity. Ideal tool to describe a fractal is the fractal dimension (FD). Fractal dimension is a shape index and particularly it can be defined as a measure of irregularly shaped objects. Irregularity in shape and behavior is shared by all of the biological, including anatomical, systems. In Euclidian geometry a number of dimension is 


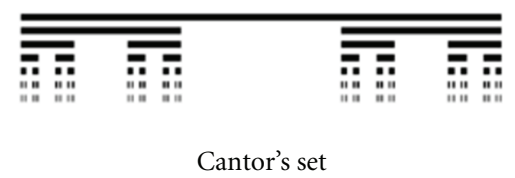

Cantor's set

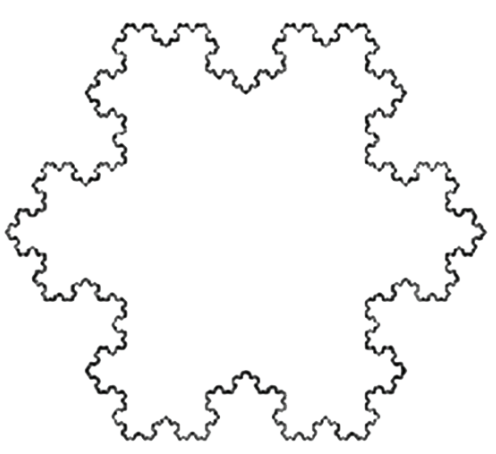

Koch's snowflake

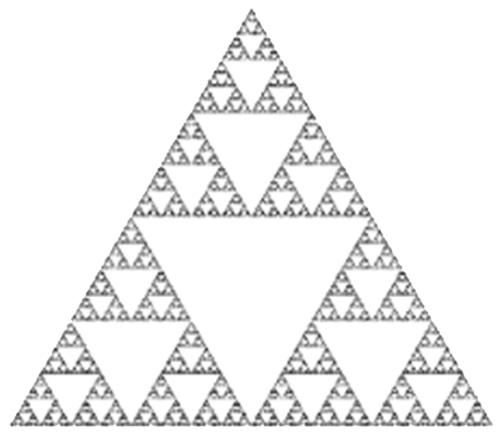

Sierpinski triangle

FiguRe 1

integer, for example, 0 is a number of dimension of a point, one number characterizes line, two dimensions built a surface, and finally three dimensions create a space. Fractal is a mathematical form where the dimension value is in the range between 0 up to 3 without $0,1,2,3$. Fractal is intermediate form between point and three-dimensional object. The lower the value of FD the more regular is the shape.

Photodynamic therapy (PDT) is very well known as the method of treating both cancer and noncancer lesions. It comprises two main agents: a light and a photosensitizer. Photosensitizer under the influence of light may induce many photochemical reactions in cells which lead to apoptosis, necrosis and autophagy [6-8]. There are many modifications of PDT which may increase its efficiency, for instance, electroporation, microdermabrasion or encapsulation into liposomes [9-12]. All above-mentioned modifications increase accumulation of photosensitizer in tissues. Other way to modify could be the combination of PDT with cysteine protease inhibitors (CPI) [13]. Cysteine proteases are the family of enzymes which are involved into the process of dissemination of malignant tumors [14].

The main aim of this study was to evaluate the influence of PDT with CPI on the number and morphology of blood vessels and increase of effectiveness of combined therapy in contrast to PDT and CPI used separately. In order to check this assumption we used the fractal dimension analysis. We applied immunohistochemistry because the colorful antibody-antigen reactions improve visualization of the blood vessels as compared to standard HE staining. We also aimed at evaluating whether the PDT combined with CPI may increase the depth of tumor necrosis and survival time of animals.

\section{Materials and Methods}

2.1. Animals. One hundred and twenty female Wistar rats (medium weight-200 g) in the age of 3 up to 3,5 months were divided into four groups (30 rats in each group). Experiments were approved by the Local Ethics Committee in Wroclaw.

2.2. Tumor. Mammary solid adenocarcinoma (Adenocarcinoma solidum mammae) was obtained from the Institute of
Oncology in Gliwice, Poland, and this was implanted as the suspension under the skin in volume $1 \mathrm{~cm}^{3}$ of tumor cells in $0,9 \% \mathrm{NaCl}$. The site of that implantation was left abdominal region.

2.3. Photosensitizer. Chlorin e6 (Frontier Scientific, Inc., USA) was obtained in the crystal form and this was dissolved in $0,9 \% \mathrm{NaCl}$ alkalized by $0,05 \mathrm{M} \mathrm{NaOH}$. Photosensitizer was applied into peritoneum in the dose $10 \mathrm{mg} / \mathrm{kg}$ of body weight.

2.4. Cysteine Protease Inhibitors (CPI). CPI was a kind gift from dr Y. Saleh of the Department of Biochemistry, Wroclaw Medical University. CPI were extracted from homogenate of human placenta. Inhibitor was purified by chromatographic methods. Purified and sterilized inhibitor was dissolved in $0,9 \% \mathrm{NaCl}$ and applied subcutaneously in $500 \mu \mathrm{g}$ dose per rat.

2.5. Immunohistochemistry. After excision the tumors were fixed in $4 \%$ formalin, embedded in paraffin blocks, and, using microtome cut in $5 \mu \mathrm{m}$ thick slices, stained using anti-VEGF antibody (Sigma, USA) and anti-bFGF antibody (Sigma, USA). Visualization of these growth factors was achieved using Vectastain ABC Kit (Vector Laboratories, Burlingame, CA, USA) and following instructions received from the supplier of the kit. In our study we used 1:50 concentration for VEGF and bFGF. The expression of VEGF and bFGF was estimated in endothelial cells of blood vessels by two independent pathologists.

2.6. Source of Light. Irradiation was performed using halogen lamp, Penta Lamps (Teclas, Switzerland) using built-in band filter at the wavelength $654 \pm 20 \mathrm{~nm}$ and total energy dose $-150 \mathrm{~J} / \mathrm{cm}^{2}$.

2.7. Treatment Protocols. Control group-first day: tumor implantation; fifth day: measurement of tumor necrosis.

Group which was treated using PDT (PDT) - first day: tumor implantation, second day: application of chlorin e6 in 
the dose of $10 \mathrm{mg} / \mathrm{kg}$ of body weight, third day: irradiation, fifth day: measurement of tumor necrosis.

Group which was treated using CPI (CPI) - first day: tumor implantation, second day: application of cystein protease inhibitors in $500 \mu \mathrm{g}$ dose per animal, and fifth day: measurement of tumor necrosis.

Group which was treated using PDT and CPI together (PDT+CPI) - first day: tumor implantation, second day: application of cystein protease inhibitors in $500 \mu \mathrm{g}$ dose per animal, third day: application of chlorin e6 in the dose of $10 \mathrm{mg} / \mathrm{kg}$ of body weight, fourth day: irradiation, and fifth day: measurement of tumor necrosis.

On the fifth day, each group was divided into two equal subgroups. In the first subgroup animals were sacrificed using the lethal dose of bioketan. During the autopsy the samples from the tumor, lungs, liver, and kidneys were taken to microscopic examination.

Animals from the second subgroup were kept in order to estimate the time of survival. In case of death of any animal the autopsy was performed using the same protocol as in the first subgroup.

2.8. Measurement of Necrosis Depth. Microscopic slides were stained using standard hematoxylin-eosin method and next they were evaluated in light microscope (Olympus BX40) in 200x magnification. Depth of necrosis was measured using an eye-piece graticule.

2.9. Evaluation of the Number of Blood Vessels. Number of blood vessels was counted on the microscopic glass slides stained using anti-bFGF and anti-VEGF antibodies. We chose randomly slides with 10 tumors (from 10 animals) from each group. We counted the number of blood vessels in five fields of view at the magnification 400x and next we calculated the average number from these five fields.

2.10. Statistical Analysis. Every statistical analysis was performed using Statistica version 6.0 (StatSoft Inc.). Hypotheses were verified on 0,05 significance level. Due to lack of normal distribution we used nonparametric KolmogorovSmirnov test.

2.11. Fractal Dimension Analysis (FDA). We used computer program Fractalyse version 2.4 (http://www.fractalyse.org/). Any graphic operation was performed in GIMP version 2.4.7. Fractalyse software enables measuring fractal dimension using box-counting method. Fractal dimension $\left(D_{S}\right)$ is counted using following formula [15]:

$$
D_{S}=\lim _{\varepsilon \rightarrow 0} \frac{\log N(\varepsilon)}{\log (1 / \varepsilon)},
$$

where $D_{S}$ - fractal dimension, $\varepsilon$-length of box which creates mesh covering surface with examining pattern, $N(\varepsilon)$ minimal number of boxes which are required to cover examining pattern.

Microscopic slides which were stained using anti-VEGF or anti-bFGF antibodies were examined using 100x magnification and the micrographs of blood vessels were taken in

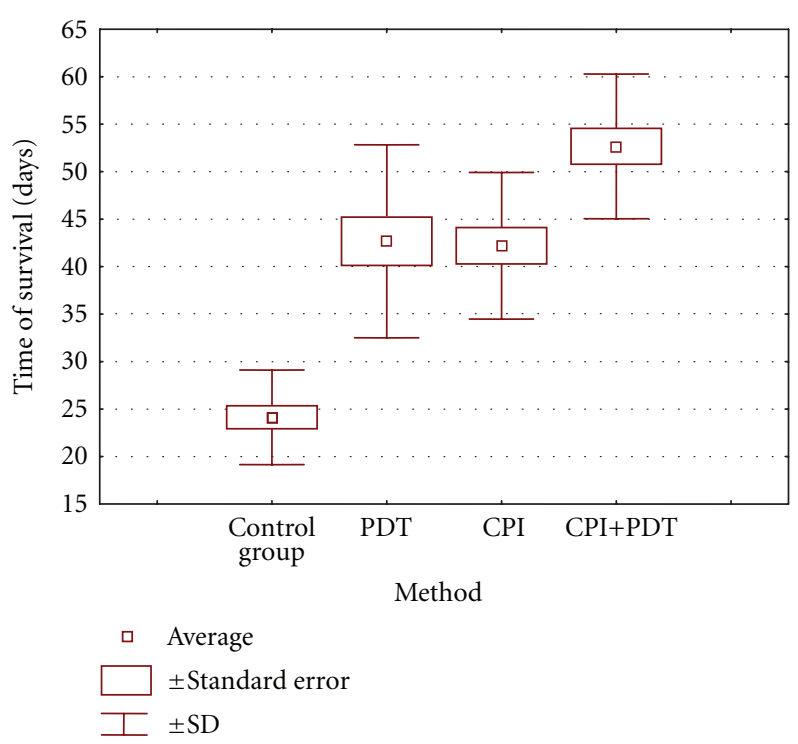

Figure 2: Influence of various treatment methods on time of survival (SD—standard deviation).

$1280 \times 1024$ points resolution. Next, the micrographs were converted into the binary white and black format. We applied Laplace filter using matrix shown below:

$$
\left|\begin{array}{ccc}
-1 & -1 & -1 \\
-1 & 8 & -1 \\
-1 & -1 & -1
\end{array}\right| \text {. }
$$

It was necessary to use the Laplace filter because this filter is able to generate very precise and thin outlines of blood vessels [16]. After all these procedures the micrographs of blood vessels were entered to Fractalyse program for estimating the fractal dimension.

\section{Results}

3.1. Influence of Various Treatment Methods on Time of Survival. Figure 2 shows the average time of survival in each group. Average survival time in control group was 24 days. The longest survival time was observed in the group which was treated by PDT and CPI together, that is, 53 days. The groups of animals which were treated using only one method (PDT or CPI separately) were characterized by similar survival time, that is, 43 days. The average survival time in every group was statistically different in comparison to control group.

Table 1 shows percentage of completely cured (complete responses) animals in each group. The animals were considered as completely cured when the survival time was 65 days or longer and no palpable tumor was found. The highest percentage of cure rate was observed in PDT+CPI group, that is, $33,33 \%$. In the PDT group it reached $13,33 \%$, in CPI group that percentage was only $6,67 \%$. In control group none of animal survived 65 days or more. 
TABle 1: Percentage of complete responses in animals treated with different methods and in the control group.

\begin{tabular}{lcccc}
\hline & Control group & PDT & CPI & CPI+PDT \\
\hline $\begin{array}{l}\text { Percentage of } \\
\text { complete responses }\end{array}$ & $0,00 \%$ & $13,33 \%$ & $6,67 \%$ & $33,33 \%$ \\
\hline
\end{tabular}

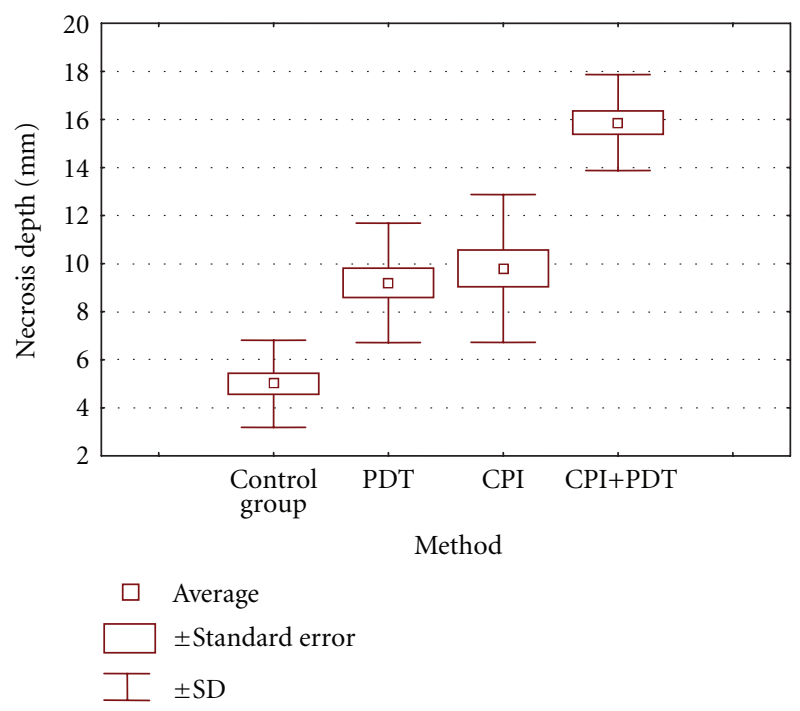

FIGURE 3: Influence of various treatment methods on necrosis depth (SD—standard deviation).

3.2. Influence of Various Treatment Methods on Necrosis Depth. Figure 3 shows the average depth of necrosis within the tumor. The lowest value was observed in control group $(5 \mathrm{~mm})$. Separate application of PDT and CPI caused increase of necrosis depth (for PDT 9,20 $\mathrm{mm}$ and 9,80 $\mathrm{mm}$ for CPI). These values were significantly different in comparison to the control group. There was no statistical difference between the groups in which PDT and CPI were applied separately. The highest value $(15,87 \mathrm{~mm})$ of necrosis depth was noted in the group which was treated by PDT and CPI together.

\subsection{Influence of Various Treatment Methods on the Value of} Vessels' Fractal Dimension. Figure 4 shows value of FD of blood vessels. The higher value was noted in control group, that is, 1,135 while the lowest value was observed in the group which was treated by cystein protease inhibitors $(0,933)$. The differences between FD in comparison to control group were observed in CPI group and PDT+CPI group $(0,982)$. The fractal dimension in PDT group was 1,037.

Table 2 shows the average number of vessels versus fractal dimension analysis. The lowest number of vessels was seen in control group (10 for VEGF and 8 for bFGF). Higher numbers of vessels were observed in the PDT group (17 for VEGF and 15 for bFGF) and in group PDT+CPI (18 for VEGF and 12 for bFGF).

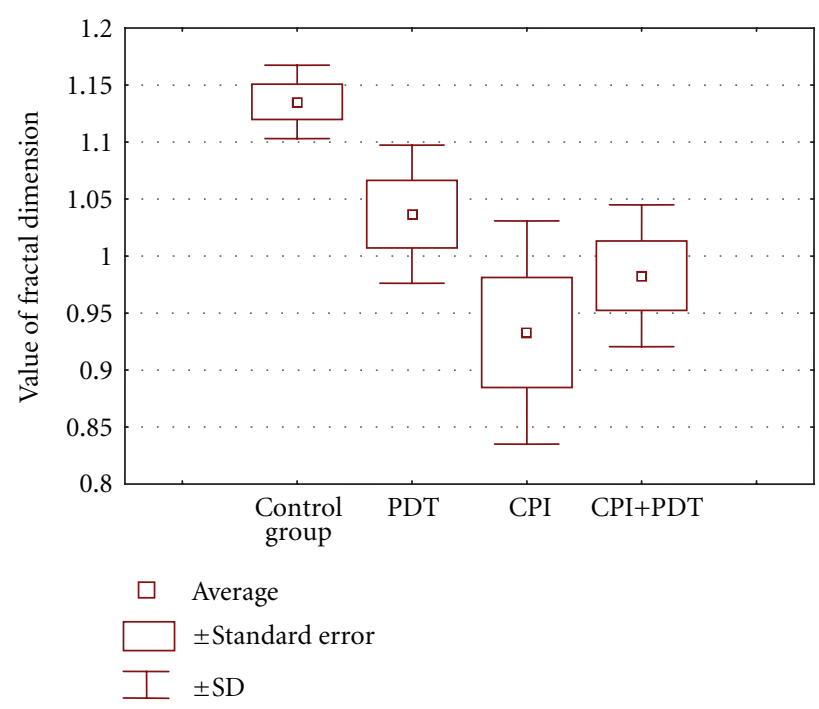

FIGURE 4: Influence of various treatment methods on the value of vessels' fractal dimension ( $\mathrm{SD}$ — standard deviation).

TABLE 2: The average number of blood vessels versus fractal dimension analysis in groups of animals treated with different methods and in the control group.

\begin{tabular}{lcccc}
\hline \multicolumn{4}{c}{ Average number of vessels } & \\
& Control group & PDT & CPI & PDT+CPI \\
\hline VEGF & 10 & 17 & 11 & 18 \\
bFGF & 8 & 15 & 8 & 12 \\
Fractal dimension & 1,135 & 1,037 & 0,933 & 0,982 \\
\hline
\end{tabular}

\section{Discussion}

Our results revealed that photodynamic therapy combined with cystein protease inhibitors causes increase of effectiveness of treatment in comparison to other methods. Higher effectiveness of PDT with CPI results in longer survival time of animals and greater depth of necrosis in the tumors. The attempts of combining the photodynamic therapy with cystein protease inhibitors seem to be reasonable and the positive synergistic effects were observed in our previous experiments [13].

The whole mechanism of photodynamic therapy is not clear. Next to destruction of cells due to phototoxic reactions, PDT influences blood vessels. Blood vessels inside the tumor are very important target on the way to tumor destruction. Many studies revealed that the time between application of photosensitizer and irradiation plays a key role in that damage. In case of rat chondrosarcoma, significant changes within vessels were achieved in the group which was irradiated 5 and 30 minutes after intravenous application of benzoporphyrin derivative. In a group which was irradiated after 180 minutes pathological lesions within vessels were less intensive [17]. Blood vessel network is characterized by highly complex spatial arrangement. Euclidean geometry because of its applicability to linear and regular forms, should not be applied to biological objects that one of 
their most important feature is the irregularity in shape. Vascular network may be roughly considered as fractal. Most of the natural shapes are similar to fractals. Trees, clouds and neuronal network are good examples. Fractal dimension is a very useful parameter in describing such shapes. Many studies revealed significant differences in value of FD between normal and tumor tissue or low grade versus high grade tumors. The example of such dependence is the difference between vascular average FD of low-grade renal carcinoma $\left(D_{s}=1,55\right)$ and highgrade $\left(D_{s}=1,45\right)[18]$. In case of renal carcinoma characterized by higher intensity of necrosis $D_{s}=1,38$ versus $D_{s}=1,52$ in tumors without necrosis. In case of liver tumors an average value of FD is 1,62 in comparison to surrounding tissue in which $D_{s}=1,47$ [18]. The results showed above indicate that the tumor vessel network is more chaotic than in surrounding tissue.

Our results showed that the highest value of FD was found in control group, which may suggest that the network of blood vessels is here more complex than in other groups. Application of PDT, CPI, and both methods together leads to decrease of fractal dimension. The lowest value of FD was observed in PDT+CPI group, thus showing that the combined therapy results in less chaotic arrangement of the blood vessels. The comparison of FD with quantity of blood vessels revealed that the shape of blood vessels was changed into more regular but amount of vessels was similar. The similar conclusions appeared after the comparison of number of vessels in PDT and PDT+CPI group. In both mentioned groups the number of vessels was increased and accompanied by the reduction of FD value. The general increase of blood vessel number in PDT+CPI group may suggest intensification of angiogenesis. Angiogenesis may be a result of the feedback as a response to destruction of blood vessels during PDT and to the increase in secretion of VEGF and bFGF [3-5]. The increase of vessel number next to reduction of fractal dimension proves that diameter of vessels decreases (the lower fractal dimension-near to 0 the shape more similar to point which may be interpreted as a reduction of diameter).

Fractal dimension analysis of blood vessels may be a very efficient tool which enables comparison of shape and distribution of vessels on the surface. FD is widely used analysis of shapes in various visualization techniques for instance X-rays photos, ultrasonography and computed tomography [19-21]. Analysis of fractal dimension requires procedures which enable separation of examined shape and background. In case of microscopic slides stained using the routine hematoxylin-eosin method the dynamics of colors is poor which may make difficulties in automatic separation of examined shapes. Computer systems require strong contrast images (very useful is the staining against CD34 in case of analysis of blood vessels) and special algorithms to search out examined shapes are also needed [2].

\section{Conclusions}

Our results revealed that fractal dimension analysis is a very useful tool in estimating differences between irregular shapes like blood vessels in PDT-treated tumors. Thus, the implementation of FDA algorithms could be useful method in evaluating the efficacy of PDT of tumors.

\section{References}

[1] P. Birner, A. Obermair, M. Schindl, H. Kowalski, G. Breitenecker, and G. Oberhuber, "Selective immunohistochemical staining of blood and lymphatic vessels reveals independent prognostic influence of blood and lymphatic vessel invasion in early-stage cervical cancer," Clinical Cancer Research, vol. 7, no. 1, pp. 93-97, 2001.

[2] A. di Leva, F. Grizzi, G. Ceva-Grimaldi et al., "Fractal dimension as a quantitator of the microvasculature of normal and adenomatous pituitary tissue," Journal of Anatomy, vol. 211, no. 5, pp. 673-680, 2007.

[3] D. Dabkeviciene, A. Sasnauskiene, E. Leman et al., "MTHPCmediated photodynamic treatment up-regulates the cytokines VEGF and IL-1alpha," Photochemistry and Photobiology, vol. 88, no. 2, pp. 432-439, 2012.

[4] H. Nakagawa, T. Matsumiya, H. Sakaki et al., "Expression of vascular endothelial growth factor by photodynamic therapy with mono-l-aspartyl chlorin e6 (NPe6) in oral squamous cell carcinoma," Oral Oncology, vol. 43, no. 6, pp. 544-550, 2007.

[5] J. Yang, A. C. Chen, Q. Wu et al., "The influence of temperature on 5-aminolevulinic acid-based photodynamic reaction in keratinocytes in vitro," Photodermatology, Photoimmunology \& Photomedicine, vol. 26, no. 2, pp. 83-88, 2010.

[6] C. M. N. Yow, C. K. Wong, Z. Huang, and R. J. Ho, "Study of the efficacy and mechanism of ALA-mediated photodynamic therapy on human hepatocellular carcinoma cell," Liver International, vol. 27, no. 2, pp. 201-208, 2007.

[7] A. Radestock, P. Elsner, B. Gitter, and U. C. Hipler, "Induction of apoptosis in HaCaT cells by photodynamic therapy with chlorin e6 or pheophorbide a," Skin Pharmacology and Physiology, vol. 20, no. 1, pp. 3-9, 2006.

[8] P. Harrod-Kim, "Tumor ablation with photodynamic therapy: introduction to mechanism and clinical applications," Journal of Vascular and Interventional Radiology, vol. 17, no. 9, pp. 1441-1448, 2006.

[9] J. Saczko, M. Nowak, N. Skolucka, J. Kulbacka, and M. Kotulska, "The effects of the electro-photodynamic in vitro treatment on human lung adenocarcinoma cells," Bioelectrochemistry, vol. 79, no. 1, pp. 90-94, 2010.

[10] M. J. P. Gerritsen, T. Smits, M. M. Kleinpenning, P. C. M. Van De Kerkhof, and P. E. J. Van Erp, "Pretreatment to enhance protoporphyrin IX accumulation in photodynamic therapy," Dermatology, vol. 218, no. 3, pp. 193-202, 2009.

[11] B. Osiecka, K. Jurczyszyn, K. Symonowicz et al., "In vitro and in vivo matrix metalloproteinase expression after photodynamic therapy with a liposomal formulation of aminolevulinic acid and its methyl ester," Cellular and Molecular Biology Letters, vol. 15, no. 4, pp. 630-650, 2010.

[12] M. S. T. Rocha, C. M. Lucci, J. P. F. Longo et al., "Aluminumchloride-phthalocyanine encapsulated in liposomes: activity against naturally occurring dog breast cancer cells," Journal of Biomedical Nanotechnology, vol. 8, no. 2, pp. 251-257, 2012.

[13] Y. Saleh, P. Ziolkowski, M. Siewinski, J. Milach, P. Marszalik, and J. Rybka, "The combined treatment of transplantable solid mammary carcinoma in wistar rats by use of photodynamic therapy and cysteine proteinase inhibitor," In Vivo, vol. 15, no. 4, pp. 351-357, 2001. 
[14] K. Sheahan, S. Shuja, and M. J. Murnane, "Cysteine protease activities and tumor development in human colorectal carcinoma," Cancer Research, vol. 49, no. 14, pp. 3809-3814, 1989.

[15] F. Grizzi, C. Russo, P. Colombo et al., "Quantitative evaluation and modeling of two-dimensional neovascular network complexity: the surface fractal dimension," BMC Cancer, vol. 5, article 14, 2005.

[16] H. Ahammer and T. T. J. DeVaney, "The influence of edge detection algorithms on the estimation of the fractal dimension of binary digital images," Chaos, vol. 14, no. 1, pp. 183-188, 2004.

[17] V. H. Fingar, P. K. Kik, P. S. Haydon et al., "Analysis of acute vascular damage after photodynamic therapy using benzoporphyrin derivative (BPD)," British Journal of Cancer, vol. 79, no. 11-12, pp. 1702-1708, 1999.

[18] F. Grizzi, B. Franceschini, B. Fiamengo, C. Russo, and N. Dioguardi, "Vascular architecture: is it a helpful histopathological biomarker for hepatocellular carcinoma?" Journal of Zhejiang University. Science. B, vol. 8, no. 4, pp. 217-220, 2007.

[19] M. E. Mavroforakis, H. V. Georgiou, N. Dimitropoulos, D. Cavouras, and S. Theodoridis, "Mammographic masses characterization based on localized texture and dataset fractal analysis using linear, neural and support vector machine classifiers," Artificial Intelligence in Medicine, vol. 37, no. 2, pp. 145-162, 2006.

[20] R. M. Rangayyan and T. M. Nguyen, "Fractal analysis of contours of breast masses in mammograms," Journal of Digital Imaging, vol. 20, no. 3, pp. 223-237, 2007.

[21] T. Chikui, K. Tokumori, K. Yoshiura, K. Oobu, S. Nakamura, and K. Nakamura, "Sonographic texture characterization of salivary gland tumors by fractal analyses," Ultrasound in Medicine and Biology, vol. 31, no. 10, pp. 1297-1304, 2005. 


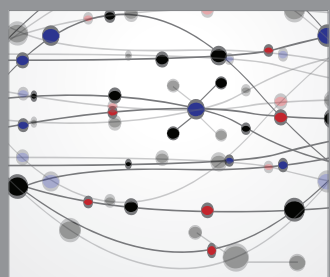

The Scientific World Journal
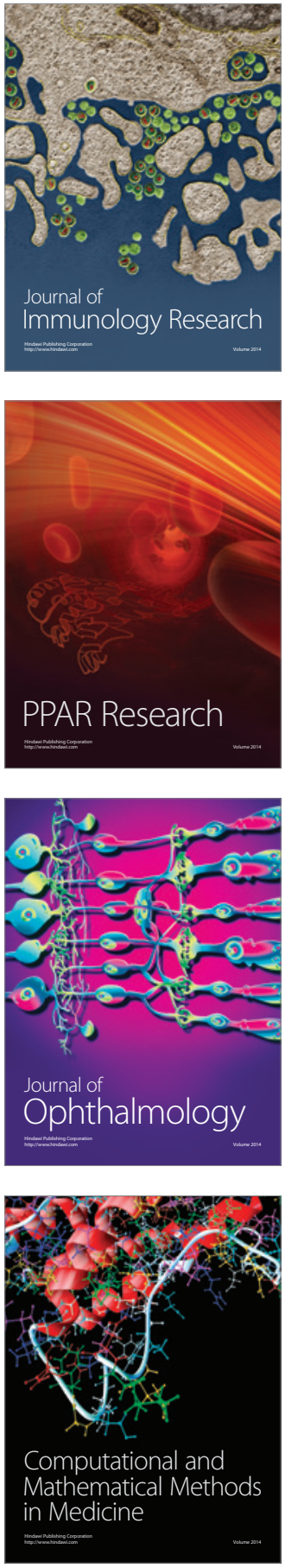

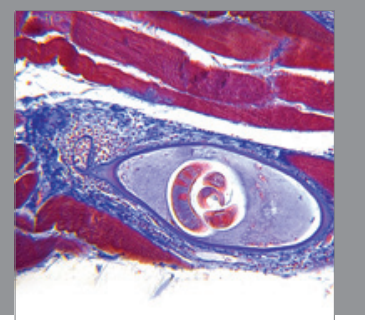

Gastroenterology

Research and Practice
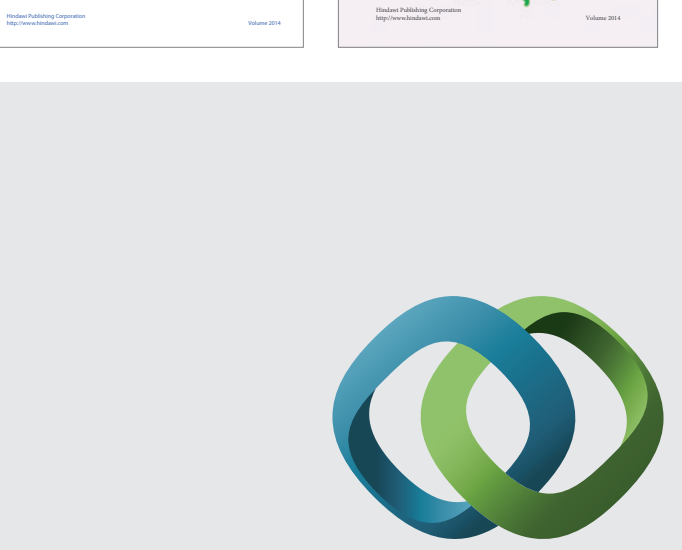

\section{Hindawi}

Submit your manuscripts at

http://www.hindawi.com
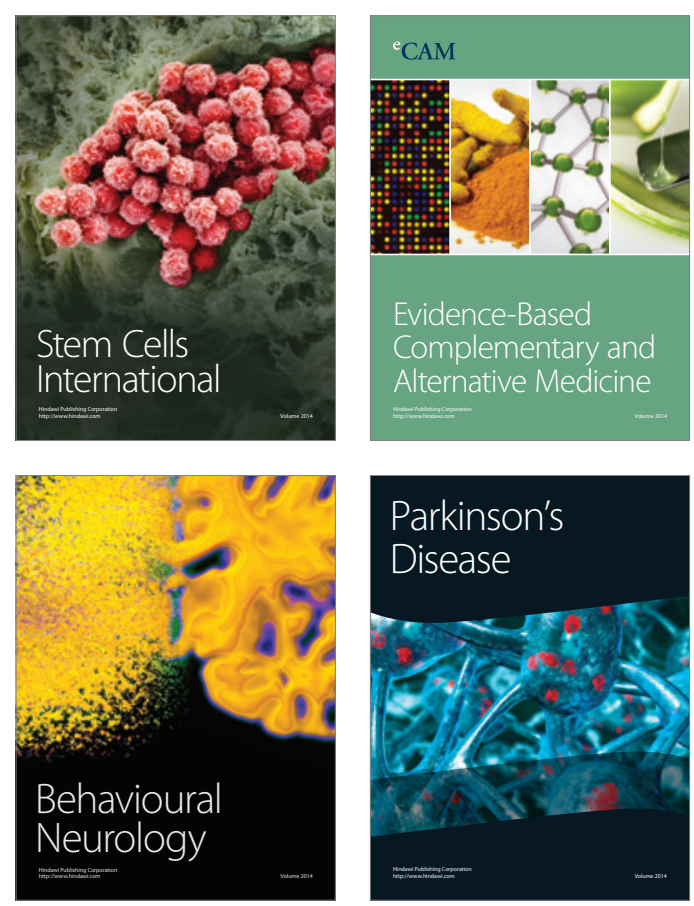

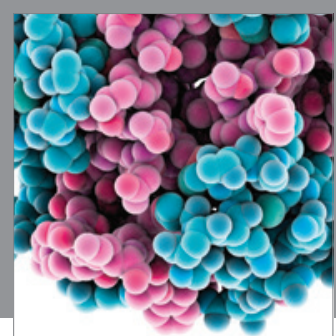

Journal of
Diabetes Research

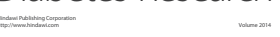

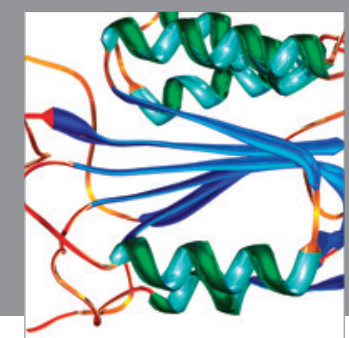

Disease Markers
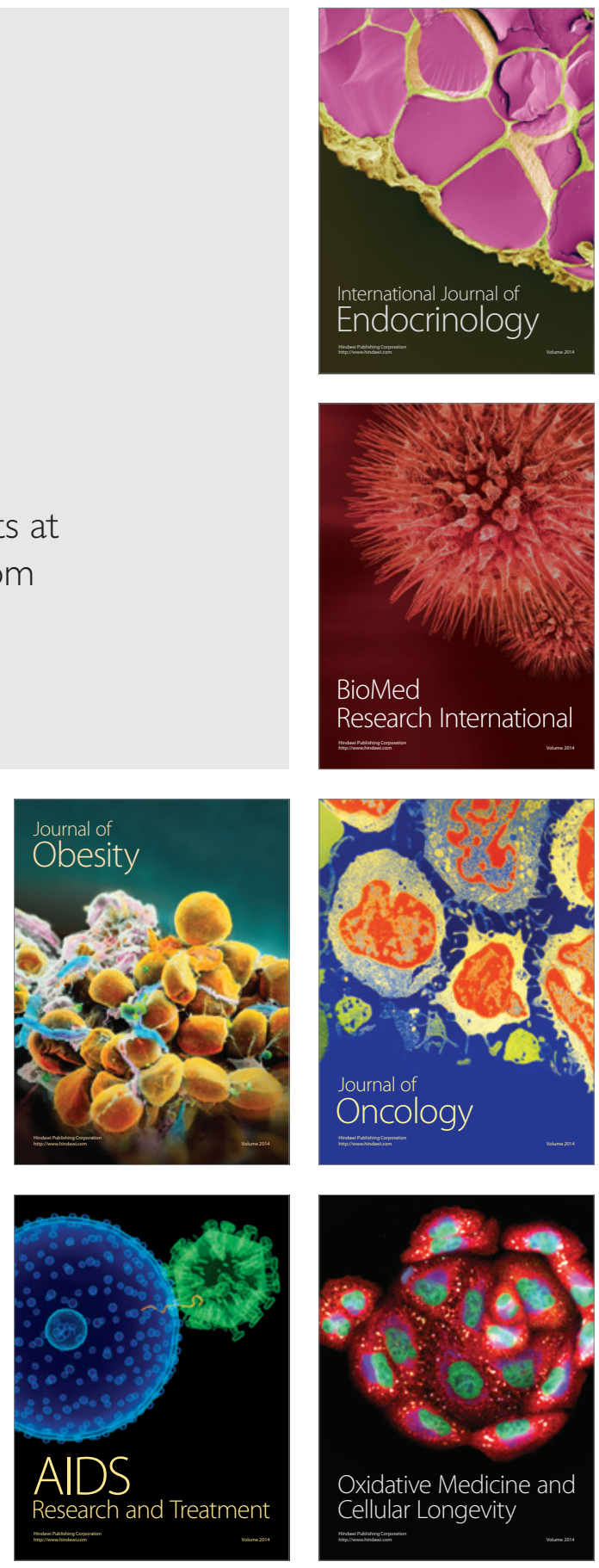